Supporting Information for

\title{
Three-Carbon Ring Expansion by Cyclopropane Insertion: Macrocyclic Musks from Readily Available C-12 Starting Materials
}

\author{
Georg Rüedi, ${ }^{*}$ Matthias Nagel, ${ }^{\dagger}$ and Hans-Jürgen Hansen \\ Organisch-chemisches Institut, University of Zürich, Winterthurerstrasse 190, CH-8057 Zürich, Switzerland
}

\section{General Details}

Flash chromatography: silica gel 60 (Merk), grain size 0.040-0.063 mm. IR Spectra: Perkin-Elmer 1600 Series FT-IR spectrometer. NMR Spectra: Bruker ARX-300 (300/75 MHz) and Avance DRX 600 (600/150 $\mathrm{MHz})$; chemical shifts $(\delta, \mathrm{ppm})$ relative to $\mathrm{CD}(\mathrm{H}) \mathrm{Cl}_{3}(7.27 / 77.00 \mathrm{ppm})$; for complete assignments of ${ }^{1} \mathrm{H}$ NMR signals COSY, TOCSY, NOESY, ROESY 2D- or 1D-NMR methods were applied; for complete assignments of ${ }^{13} \mathrm{C}$ NMR signals HMBC, HSQC, and HSQC-TOCSY 2D-NMR methods were employed. GC/MS: Hewlett Packard HP-5971 Series (mass-selective detector; EI, 70 eV).

\section{General procedure for the addition of Grignard reagents to cycloalk-3-en-}

\section{1-ones.}

A freshly prepared solution of the corresponding magnesium bromide (42 mmol) in dry THF (80 $\mathrm{mL})$ was added at $0{ }^{\circ} \mathrm{C}$ to a suspension of the substrate ketone $(30 \mathrm{mmol})$ and $\mathrm{CeCl}_{3}(30 \mathrm{mmol})$ in dry THF (75 $\mathrm{mL}$ ). The resulting mixture was allowed to warm to room temperature within $30 \mathrm{~min}$ and was subsequently quenched with saturated $\mathrm{NH}_{4} \mathrm{Cl}(150 \mathrm{~mL})$ and then poured into a separatory funnel containing ether (200 $\mathrm{mL})$. The organic layer was separated, and the aqueous phase extracted with ether $(3 \times 150 \mathrm{~mL})$. The combined organic layers were washed with saturated $\mathrm{NH}_{4} \mathrm{Cl}$ and brine, dried over $\mathrm{MgSO}_{4}$, filtered and concentrated under reduced pressure. The residue was purified by flash chromatography on silica gel. An analytical sample was distilled (Kugelrohr).

\section{(E)-1-Cyclopropylcyclododec-3-enol (10)}

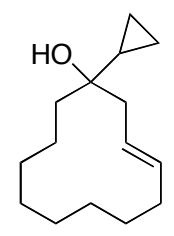

The reaction of $(E)-3$-cyclododecenone $(6.0 \mathrm{~g}, 33.3 \mathrm{mmol})$ with cyclopropyl magnesium bromide following the general procedure afforded $7.3 \mathrm{~g}(98 \%)$ of the title compound as a colorless oil.

IR (film) 3445, 3083, 2925, 2851, 1462, 1444, 1349, 1299, 1197, $1144 \mathrm{~cm}^{-1} ;{ }^{1} \mathrm{H}$ NMR (300 $\left.\mathrm{MHz}_{2} \mathrm{CDCl}_{3}\right) \delta$ 5.65-5.45 (m, 2H), 2.24-2.21 (m, 2H), 2.14-2.07 (m, 2H), 1.61-1.26 (m, 15H), $1.01-0.86(\mathrm{~m}, 1 \mathrm{H}), 0.44-0.28(\mathrm{~m}, 4 \mathrm{H}) ;{ }^{13} \mathrm{C} \mathrm{NMR}\left(75 \mathrm{MHz}, \mathrm{CDCl}_{3}\right) \delta 134.5,125.6,73.0,43.1,37.3,33.3$, 28.5, 27.1, 26.1 , 25.2, 24.4, 20.5, 19.1, 0.3, -0.4; MS m/z (rel intensity) 222 (3), 151 (12), 137 (9), 123 (8), 109 (8), 97 (45), 84 (68), 69 (100). Anal. Calcd for $\mathrm{C}_{15} \mathrm{H}_{26} \mathrm{O}$ (222.37): C, 81.02; H, 11.78. Found: C, $80.89 ; \mathrm{H}, 11.91$. 


\section{(E)-1-Cyclopropylcyclotridec-3-enol}

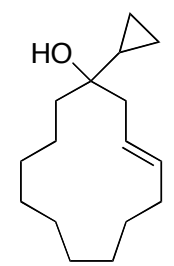

The reaction of $(E)-3$-cyclotridecenone ${ }^{1}(2.5 \mathrm{~g}, 12.9 \mathrm{mmol})$ with cyclopropyl magnesium bromide following the general procedure afforded $3.0 \mathrm{~g}(99 \%)$ of the title compound as a colorless oil.

IR (film) 3446, 3083, 3004, 2931, 2858, 1461, 1445, 1059, $971 \mathrm{~cm}^{-1} ;{ }^{1} \mathrm{H}$ NMR $(300 \mathrm{MHz}$, $\left.\mathrm{CDCl}_{3}\right) \delta 5.53-5.33(\mathrm{~m}, 2 \mathrm{H}), 2.18(\mathrm{~d}, J=6.6 \mathrm{~Hz}, 2 \mathrm{H}), 2.07-1.89(\mathrm{~m}, 2 \mathrm{H}), 1.62-1.20(\mathrm{~m}, 17 \mathrm{H})$, $1.02-0.85(\mathrm{~m}, 1 \mathrm{H}), 0.40-0.28(\mathrm{~m}, 4 \mathrm{H}) ;{ }^{13} \mathrm{C} \mathrm{NMR}\left(75 \mathrm{MHz}, \mathrm{CDCl}_{3}\right) \delta 134.4,126.2,72.6,43.1,38.5,32.3$, 27.7, 27.6, 27.4, 25.1, 24.9, 24.6, 20.5, 19.3, 0.2, -0.1; MS m/z (rel intensity) 236 (1), 218 (2), 151 (10), 137 (7), 123 (8), 97 (51), 84 (73), 69 (100). Anal. Calcd for $\mathrm{C}_{16} \mathrm{H}_{28} \mathrm{O}$ (236.40): C, 81.29; H, 11.93. Found: C, 81.42; H, 11.99 .

\section{(Z)-1-Cyclopropylcyclooct-3-enol}

HO $\triangle$ The reaction of $(Z)-3$-cyclooctenone ${ }^{2,3}(3.5 \mathrm{~g}, 28.2 \mathrm{mmol})$ with cyclopropyl magnesium bromide following the general procedure afforded $4.1 \mathrm{~g}(87 \%)$ of the title compound as a colorless oil.

IR (film) 3442, 3082, 3016, 2928, 2857, 1464, 1446, 1050, 1002, 942, $771 \mathrm{~cm}^{-1}$; ${ }^{1} \mathrm{H}$ NMR $(300 \mathrm{MHz}$, $\left.\mathrm{CDCl}_{3}\right) \delta 5.81-5.52(\mathrm{~m}, 2 \mathrm{H}), 2.35(\mathrm{dd}, J=12.9,8.4 \mathrm{~Hz}, 1 \mathrm{H}), 2.23-2.10(\mathrm{~m}, 3 \mathrm{H}), 1.64-1.39(\mathrm{~m}, 7 \mathrm{H})$, $1.00-0.91(\mathrm{~m}, 1 \mathrm{H}), 0.40-0.27(\mathrm{~m}, 4 \mathrm{H}) ;{ }^{13} \mathrm{C} \mathrm{NMR}\left(75 \mathrm{MHz}, \mathrm{CDCl}_{3}\right.$ ) $\delta 132.4,127.1,74.7,38.5,37.1,29.4$, 26.5, 22.3, 20.4, 0.1, -0.1; MS m/z (rel intensity) 166 (3), 137 (7), 123 (10), 111 (12), 97 (81), 84 (40), 69 (100). HRMS (EI) calcd for $\mathrm{C}_{11} \mathrm{H}_{18} \mathrm{O} \mathrm{m} / z$. 166.1358, found $\mathrm{m} / \mathrm{z}, 166.1358$.

\section{(E)-1-Isopropenylcyclododec-3-enol (9d)}

The reaction of $(E)-3$-cyclododecenone $(3.0 \mathrm{~g}, 16.6 \mathrm{mmol})$ with isopropenyl magnesium
bromide following the general procedure afforded $3.6 \mathrm{~g}(96 \%)$ of the title compound as a
colorless oil.
IR (film) $3398,2926,2852,1462,1446,1372,1120 \mathrm{~cm}^{-1} ;{ }^{1} \mathrm{H} \mathrm{NMR}\left(300 \mathrm{MHz}, \mathrm{CDCl}_{3}\right) \delta$ 5.66-5.47 (m, 2H), $4.92(\mathrm{~s}, 1 \mathrm{H}), 4.85-4.83(\mathrm{~m}, 1 \mathrm{H}), 2.53(\mathrm{dd}, J=14.5,8.1 \mathrm{~Hz}, 1 \mathrm{H}), 2.26(\mathrm{dd}, J=14.5,5.4$ $\mathrm{Hz}, 1 \mathrm{H}), 2.12-2.01(\mathrm{~m}, 2 \mathrm{H}), 1.78-1.21(\mathrm{~m}, 18 \mathrm{H}) ;{ }^{13} \mathrm{C} \mathrm{NMR}\left(75 \mathrm{MHz}, \mathrm{CDCl}_{3}\right) \delta 150.3,134.7,125.7$, 110.2 , 76.7, 41.4, 34.8, 33.2, 28.3, 27.0, 25.5, 24.9, 24.5, 19.3, 18.8; MS m/z (rel intensity) 222 (3), 179 (5), 151 (4), 137 (9), 123 (12), 109 (14), 97 (39), 84 (56), 69 (100). HRMS (EI) calcd for $\mathrm{C}_{15} \mathrm{H}_{26} \mathrm{O} \mathrm{m} / \mathrm{z}$ 222.1984 , found $m / z, 222.1983$.

\section{(E)-1-[(Z)-Propen-1-yl]cyclododec-3-enol (9e)}

The reaction of $(E)$-3-cyclododecenone $(3.8 \mathrm{~g}, 21.1 \mathrm{mmol})$ with $(Z)$-propen-1-yl magnesium
bromide following the general procedure afforded $4.5 \mathrm{~g}(97 \%)$ of the title compound as a
colorless oil. IR (film) 3368, 3012, 2929, 2849, 1552, 1346, 1160, $1009 \mathrm{~cm}^{-1} ;{ }^{1} \mathrm{H}$ NMR (300 MHz, $\left.\mathrm{CDCl}_{3}\right) \delta 5.61-5.38$ $(\mathrm{m}, 4 \mathrm{H}), 2.38(\mathrm{~d}, J=7.0 \mathrm{~Hz}, 2 \mathrm{H}), 2.10(\mathrm{q}, J=6.6 \mathrm{~Hz}, 2 \mathrm{H}), 1.87(\mathrm{~d}, J=5.3 \mathrm{~Hz}, 3 \mathrm{H}), 1.67-1.22(\mathrm{~m}, 15 \mathrm{H})$; 
${ }^{13} \mathrm{C}$ NMR $\left(75 \mathrm{MHz}, \mathrm{CDCl}_{3}\right) \delta 136.1,134.8,126.3,125.3,75.8,44.6,37.8,33.2,28.4,27.0,26.0,25.0$, 24.3, 19.1, 14.3; MS m/z (rel intensity) 222 (1), 179 (2), 165 (2), 151 (4), 137 (4), 123 (6), 109 (6), 97 (33), 84 (42), 69 (100). HRMS (EI) calcd for $\mathrm{C}_{15} \mathrm{H}_{26} \mathrm{O} \mathrm{m} / z$ 222.1984, found $\mathrm{m} / \mathrm{z} 222.1982$.

\section{(E)-1-[(E)-Propen-1-yl]cyclododec-3-enol}

HO $=$ The reaction of $(E)-3$-cyclododecenone $(2.6 \mathrm{~g}, 14.4 \mathrm{mmol})$ with $(E)$-propen-1-yl magnesium bromide following the general procedure afforded $2.9 \mathrm{~g}(90 \%)$ of the title compound as a colorless oil.

IR (film) 3359, 3013, 2930, 2847, 1471, 1442, 1345, 1160, $1008 \mathrm{~cm}^{-1} ;{ }^{1} \mathrm{H}$ NMR (300 MHz, $\left.\mathrm{CDCl}_{3}\right) \delta 5.62-5.36(\mathrm{~m}, 4 \mathrm{H}), 2.38(\mathrm{~d}, J=7.1 \mathrm{~Hz}, 2 \mathrm{H}), 2.09(\mathrm{q}, J=6.6 \mathrm{~Hz}, 2 \mathrm{H}), 1.88(\mathrm{~d}, J=5.5 \mathrm{~Hz}, 3 \mathrm{H})$, 1.65-1.21 (m, 15H); $\left.{ }^{13} \mathrm{C} \mathrm{NMR} \mathrm{(75} \mathrm{MHz,} \mathrm{CDCl}_{3}\right) \delta 136.2,132.6,126.4,125.0,75.7,39.1,37.4,35.8,26.9$, 26.4, 25.5, 22.1, 21.9, 19.9, 14.0; MS m/z (rel intensity) 222 (1), 179 (1), 165 (2), 151 (3), 137 (4), 123 (5), 109 (7), 97 (36), 84 (45), 69 (100). HRMS (EI) calcd for $\mathrm{C}_{15} \mathrm{H}_{26} \mathrm{O} \mathrm{m} / \mathrm{z}$ 222.1984, found $\mathrm{m} / \mathrm{z} 222.1982$.

\section{(E)-1-(2-Methylpropen-1-yl)cyclododec-3-enol (9f)}

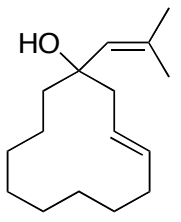

The reaction of $(E)$-3-cyclododecenone $(2.5 \mathrm{~g}, 13.9 \mathrm{mmol})$ with 2-methylpropen-1-yl magnesium bromide following the general procedure afforded $3.2 \mathrm{~g}(99 \%)$ of the title compound as a colorless oil.

IR (film) 3415, 2926, 2854, 1461, 1376, 1119, $982 \mathrm{~cm}^{-1} ;{ }^{1} \mathrm{H}$ NMR (300 MHz, $\left.\mathrm{C}_{6} \mathrm{D}_{6}\right) \delta$ 5.61-5.43 (m, 2H), 5.31-5.29 (m, 1H), $2.44(\mathrm{dd}, J=13.8,5.0 \mathrm{~Hz}, 1 \mathrm{H}), 2.34(\mathrm{dd}, J=13.1,6.6 \mathrm{~Hz}, 1 \mathrm{H})$, $2.06(\mathrm{q}, J=5.4 \mathrm{~Hz}, 2 \mathrm{H}), 1.91(\mathrm{~d}, J=1.2 \mathrm{~Hz}, 3 \mathrm{H}), 1.66(\mathrm{~d}, J=1.3 \mathrm{~Hz}, 3 \mathrm{H}), 1.86-1.22(\mathrm{~m}, 15 \mathrm{H}) ;{ }^{13} \mathrm{C}$ NMR $\left(75 \mathrm{MHz}, \mathrm{C}_{6} \mathrm{D}_{6}\right) \delta 134.4,134.3,131.5,126.4,74.7,45.4,38.5,33.5,28.7,27.3,26.5,24.8,24.4,19.5,19.0$, 18.9; MS m/z (rel intensity) 236 (1), 218 (6), 175 (3), 147 (4), 133 (7), 119 (14), 105 (17), 98 (21), 83 (100). HRMS (EI) calcd for $\mathrm{C}_{16} \mathrm{H}_{28} \mathrm{O} \mathrm{m} / z$ 236.2140, found $\mathrm{m} / z, 236.2141$.

\section{(E)-2-Methylcyclododec-3-enone}

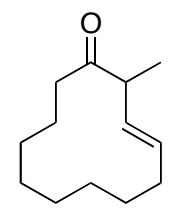

A solution of $(E)$-3-cyclododecenone $(6.0 \mathrm{~g}, 33.3 \mathrm{mmol})$ in dry DMSO (20 mL) was added dropwise to a solution of tert-BuOK $(3.7 \mathrm{~g}, 33.3 \mathrm{mmol})$ in dry DMSO $(40 \mathrm{~mL})$. The yellow solution was stirred for $15 \mathrm{~min}$ at room temperature and subsequently cooled to $0{ }^{\circ} \mathrm{C}$ at which methyl iodide $(6.1 \mathrm{~mL}, 33.3 \mathrm{mmol})$ was added in one portion. After 5 min the yellow color of the solution disappeared and stirring was continued for $1 \mathrm{~h}$ at room temperature. The mixture was quenched with saturated $\mathrm{NH}_{4} \mathrm{Cl}(150 \mathrm{~mL})$ and then poured into a separatory funnel containing ether (200 $\mathrm{mL})$. The organic layer was separated, and the aqueous phase extracted with ether $(3 \times 150 \mathrm{~mL})$. The combined organic layers were washed with water, saturated $\mathrm{NH}_{4} \mathrm{Cl}$ and brine, dried over $\mathrm{MgSO}_{4}$, filtered and concentrated under reduced pressure. The residue was purified by flash chromatography (silica gel, $5 \%$ ethyl acetate in hexanes) affording $4.0 \mathrm{~g}(62 \%)$ of the title compound as a colorless oil. An analytical sample was distilled (Kugelrohr). 
IR (film) 3011, 2932, 2860, 1707, 1463, 1366, 1196, 1046, $977 \mathrm{~cm}^{-1} ;{ }^{1} \mathrm{H}$ NMR (300 MHz, $\left.\mathrm{CDCl}_{3}\right) \delta$ 5.54-5.48 (m, 1H), 5.34-5.25 (m, 1H), 3.84-3.46 (m, 1H), 2.57 (ddd, $J=13.9,6.2,5.4 \mathrm{~Hz}, 1 \mathrm{H}), 2.31$ (ddd, $J=13.9,10.1,5.4 \mathrm{~Hz}, 1 \mathrm{H}), 1.98-1.75(\mathrm{~m}, 2 \mathrm{H}), 1.68-1.53(\mathrm{~m}, 2 \mathrm{H}), 1.47-1.15(\mathrm{~m}, 10 \mathrm{H}), 1.12(\mathrm{~d}, J=6.9$ $\mathrm{Hz}, 3 \mathrm{H}) ;{ }^{13} \mathrm{C} \mathrm{NMR}\left(75 \mathrm{MHz}, \mathrm{CDCl}_{3}\right) \delta 214.5,130.7,130.5,41.6,41.5,26.8,26.4,24.8,23.7,23.6,23.5$, 22.9, 18.4; MS m/z (rel intensity) 194 (8), 151 (4), 137 (4), 123 (5), 109 (10), 95 (26), 81 (63), 68 (100). HRMS (EI) calcd for $\mathrm{C}_{13} \mathrm{H}_{22} \mathrm{O} \mathrm{m} / z$ 194.1671, found $\mathrm{m} / \mathrm{z} 194.1669$.

\section{$\left(1 R^{*}, 2 R^{*}, 3 E\right)$-1-Cyclopropyl-2-methylcyclododec-3-enol}

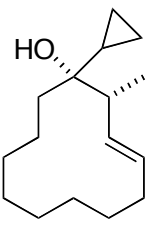

The reaction of $(E)$-2-methylcyclododec-3-enone $(2.3 \mathrm{~g}, 11.8 \mathrm{mmol})$ with cyclopropyl magnesium bromide carried out at $-30{ }^{\circ} \mathrm{C}$ (!) following the general procedure afforded $2.4 \mathrm{~g}$ $(86 \%)$ of the title compound as a colorless oil. A minor amount of the (1RS,2SR)-isomer (ca. $10 \%$ ) was evident from GC/MS analysis.

IR (film) 3665, 3082, 3005, 2926, 2850, 1460, 1446, 1382, $982 \mathrm{~cm}^{-1} ;{ }^{1} \mathrm{H}$ NMR (300 MHz, $\left.\mathrm{CDCl}_{3}\right) \delta$ 5.50-5.33 (m, 1H), 5.29-5.15 (m, 1H), 2.99-2.87 (m, 1H), 2.53-2.40 (m, 1H), 1.91-1.78 (m, 1H) $1.60-1.05(\mathrm{~m}, 14 \mathrm{H}), 1.02(\mathrm{~d}, J=6.9 \mathrm{~Hz}, 3 \mathrm{H}), 0.60-0.14(\mathrm{~m}, 5 \mathrm{H}) ;{ }^{13} \mathrm{C} \mathrm{NMR}\left(75 \mathrm{MHz}, \mathrm{CDCl}_{3}\right) \delta 132.6$, 130.0, 74.1, 39.4, 37.9, 27.0, 25.6, 24.2, 24.1, 24.0, 22.3, 18.7, 17.4, 15.4, 0.9, -0.8; MS m/z (rel intensity) 236 (2), 151 (12), 137 (7), 123 (6), 109 (8), 97 (44), 84 (49), 69 (100). HRMS (EI) calcd for $\mathrm{C}_{16} \mathrm{H}_{28} \mathrm{O} \mathrm{m} / z$ 236.2140, found $\mathrm{m} / \mathrm{z} 236.2142$.

\section{General procedure for the regioselective cyclopropanation of allylalcohols.}

To a solution of the appropriate allylalcohol $(10 \mathrm{mmol})$ in dry benzene $(100 \mathrm{~mL})$ was treated with of diethylzinc $(9.1 \mathrm{~mL}$ of $1.1 \mathrm{M}$ in toluene, $10 \mathrm{mmol})$. The resulting mixture was cooled to $5{ }^{\circ} \mathrm{C}$ at which diiodomethane $(2.0 \mathrm{~mL}, 25 \mathrm{mmol})$ was added dropwise via syringe. The progress of the reaction was monitored by TLC. After 1-3 h the mixture became cloudy and the reaction was quenched with saturated $\mathrm{NH}_{4} \mathrm{Cl}(100 \mathrm{~mL})$ and then poured into a separatory funnel containing ether $(150 \mathrm{~mL})$. The organic layer was separated, and the aqueous phase extracted with ether $(3 \times 100 \mathrm{~mL})$. The combined organic layers were washed with saturated $\mathrm{NH}_{4} \mathrm{Cl}$ and brine, dried over $\mathrm{MgSO}_{4}$, filtered and concentrated under reduced pressure. The residue was purified by flash chromatography on silica gel. An analytical sample was distilled (Kugelrohr).

\section{(E)-1-(1-Methylcyclopropyl)cyclododec-3-enol}

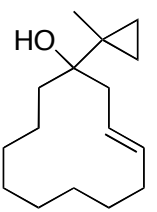

The cyclopropanation of (E)-1-isopropenylcyclododec-3-enol (9d) (3.0 g, $13.5 \mathrm{mmol})$ following the general procedure afforded $2.9 \mathrm{~g} \mathrm{(92 \% )}$ of the title compound as a colorless oil. IR (film) 3464, 3080, 3001, 2926, 2851, 1459, 1380, 1026, 1015, $982 \mathrm{~cm}^{-1} ;{ }^{1} \mathrm{H}$ NMR (300 $\left.\mathrm{MHz}, \mathrm{CDCl}_{3}\right) \delta$ 5.63-5.44 (m, 2H), 2.21-2.02 (m, 4H), 1.59-1.12 (m, 15H), 1.09 (s, 3H), $0.68-0.56(\mathrm{~m}, 2 \mathrm{H}), 0.25-0.20(\mathrm{~m}, 1 \mathrm{H}), 0.12-0.07(\mathrm{~m}, 1 \mathrm{H}) ;{ }^{13} \mathrm{C} \mathrm{NMR}\left(75 \mathrm{MHz}, \mathrm{CDCl}_{3}\right) \delta 133.8,126.2$, 75.4, 40.0, 34.9, 32.8, 27.5, 26.7, 26.2, 24.5, 24.4, 23.6, 21.3, 19.5, 10.9, 8.8; MS m/z (rel intensity) 236 (1), 
218 (2), 147 (4), 133 (7), 119 (6), 111 (19), 98 (36), 83 (100). Anal. Calcd for $\mathrm{C}_{16} \mathrm{H}_{28} \mathrm{O}(236.40)$ : C, 81.29; H, 11.93. Found: C, 81.46; H, 12.07.

\section{(E)-1-(cis-2-Methylcyclopropyl)cyclododec-3-enol}

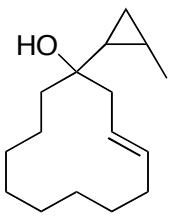

The cyclopropanation of $(E)-1-[(Z)$-propen-1-yl]cyclododec-3-enol (9e) $(3.7 \mathrm{~g}, 16.6 \mathrm{mmol})$ following the general procedure afforded $3.3 \mathrm{~g} \mathrm{(85 \% )}$ of the title compound as a colorless oil. IR (film) 3479, 3064, 2926, 2850, 1461, 1446, 1386, 1014, $981 \mathrm{~cm}^{-1}$; ${ }^{1} \mathrm{H}$ NMR (300 MHz, $\left.\mathrm{CDCl}_{3}\right) \delta 5.65-5.44(\mathrm{~m}, 2 \mathrm{H}), 2.38-2.30(\mathrm{~m}, 2 \mathrm{H}), 2.14-2.07(\mathrm{~m}, 2 \mathrm{H}), 1.65-1.24(\mathrm{~m}, 15 \mathrm{H})$, 0.96-0.78 (m, 5H), 0.60-0.49 (m, 1H), 0.32-0.15 (m, 1H); $\left.{ }^{13} \mathrm{C} \mathrm{NMR} \mathrm{(75} \mathrm{MHz,} \mathrm{CDCl}_{3}\right) \delta 134.5,125.5$, 74.2, 45.9, 37.0, 33.2, 28.4, 27.0, 26.1, 25.0, 24.3, 24.2, 19.2, 13.5, 10.4, 6.7; MS m/z (rel intensity) 236 (1), 218 (2), 165 (5), 151 (6), 137 (4), 119 (3), 111 (27), 98 (35), 83 (100). Anal. Calcd for $\mathrm{C}_{16} \mathrm{H}_{28} \mathrm{O}$ (236.40): C, 81.29; H, 11.93. Found: C, 81.25; H, 12.09.

\section{(E)-1-(trans-2-Methylcyclopropyl)cyclododec-3-enol}

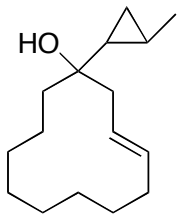

The cyclopropanation of $(E)-1-[(E)$-propen-1-yl]cyclododec-3-enol $(2.3 \mathrm{~g}, 10.3 \mathrm{mmol})$ following the general procedure afforded $1.9 \mathrm{~g}(78 \%)$ of the title compound as a colorless oil. IR (film) 3475, 3065, 2925, 2850, 1461, 1445, 1385, 1014, $981 \mathrm{~cm}^{-1}$; ${ }^{1} \mathrm{H}$ NMR (300 MHz, $\left.\mathrm{CDCl}_{3}\right) \delta 5.63-5.36(\mathrm{~m}, 2 \mathrm{H}), 2.37-2.28(\mathrm{~m}, 2 \mathrm{H}), 2.14-2.07(\mathrm{~m}, 2 \mathrm{H}), 1.66-1.19(\mathrm{~m}, 15 \mathrm{H})$, $0.98-0.80(\mathrm{~m}, 5 \mathrm{H}), 0.61-0.48(\mathrm{~m}, 1 \mathrm{H}), 0.29-0.12(\mathrm{~m}, 1 \mathrm{H}) ;{ }^{13} \mathrm{C} \mathrm{NMR}\left(75 \mathrm{MHz}, \mathrm{CDCl}_{3}\right) \delta 134.2,125.9$, 74.4, 43.3, 39.4, 31.5, 25.1, 25.0, 24.3, 24.0, 22.8, 22.5, 19.2, 14.0, 10.6, 6.4; MS m/z (rel intensity) 236 (1), 218 (2), 165 (5), 151 (5), 137 (3), 119 (3), 111 (24), 98 (43), 83 (100). Anal. Calcd for $\mathrm{C}_{16} \mathrm{H}_{28} \mathrm{O}$ (236.40): C, 81.29; H, 11.93. Found: C, 81.36; H, 12.12 .

\section{(E)-1-(2,2-Dimethylcyclopropyl)cyclododec-3-enol}

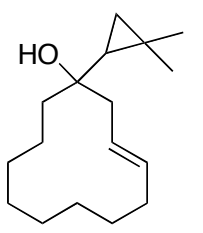

The cyclopropanation of (E)-1-(2-methylpropen-1-yl)cyclododec-3-enol (9f) (2.4 g, 10.2 mmol) using ether (!) as solvent following the general procedure afforded $1.98 \mathrm{~g}(78 \%)$ of the title compound as a colorless oil. ${ }^{4}$

IR (film) 3483, 2990, 2926, 2862, 1460, 1446, 1373, 1124, $981 \mathrm{~cm}^{-1}$; ${ }^{1} \mathrm{H}$ NMR (300 MHz, $\left.\mathrm{CDCl}_{3}\right) \delta 5.63-5.46(\mathrm{~m}, 2 \mathrm{H}), 2.36(\mathrm{~d}, J=6.5 \mathrm{~Hz}, 2 \mathrm{H}), 2.12(\mathrm{q}, J=5.0 \mathrm{~Hz}, 2 \mathrm{H}), 1.61-1.23(\mathrm{~m}, 15 \mathrm{H}), 1.27$ $(\mathrm{s}, 3 \mathrm{H}), 1.04(\mathrm{~s}, 3 \mathrm{H}), 0.69(\mathrm{dd}, J=9.2,6.3 \mathrm{~Hz}, 1 \mathrm{H}), 0.40(\mathrm{dd}, J=6.3,4.0 \mathrm{~Hz}, 1 \mathrm{H}), 0.30(\mathrm{dd}, J=9.2,4.0$ $\mathrm{Hz}, 1 \mathrm{H}) ;{ }^{13} \mathrm{C} \mathrm{NMR}\left(75 \mathrm{MHz}, \mathrm{CDCl}_{3}\right) \delta 134.2,125.9,74.1,43.1,39.3,33.2,33.1,31.5,28.9,28.3,27.0$, 26.1, 25.1, 24.3, 19.9, 19.2, 14.4; MS m/z (rel intensity) 250 (6), 232 (5), 189 (10), 161 (5), 135 (14), 125 (29), 112 (35), 97 (100). Anal. Calcd for $\mathrm{C}_{17} \mathrm{H}_{30} \mathrm{O}$ (250.42): C, 81.54; H, 12.07. Found: C, 81.44; H, 12.01 . 


\section{General procedure for silylether formation.}

A solution of the cyclopropyl alcohol $(10 \mathrm{mmol}), \mathrm{DMAP}(0.19 \mathrm{~g}, 1.5 \mathrm{mmol})$, and $\mathrm{Et}_{3} \mathrm{~N}(2.1 \mathrm{~mL}, 15 \mathrm{mmol})$ in DMF (40 mL) was cooled to $0{ }^{\circ} \mathrm{C}$ and $\mathrm{TMSCl}(1.5 \mathrm{~mL}, 12 \mathrm{mmol})$ was added via syringe. The resulting cloudy solution was stirred for $1 \mathrm{~h}$ at room temperature and then poured into a separatory funnel containing ether $(100 \mathrm{~mL})$ and saturated $\mathrm{NH}_{4} \mathrm{Cl}(100 \mathrm{~mL})$. The organic layer was separated, and the aqueous phase extracted with ether $(3 \times 100 \mathrm{~mL})$. The combined organic layers were washed with saturated $\mathrm{NH}_{4} \mathrm{Cl}$ and brine, dried over $\mathrm{MgSO}_{4}$, filtered and concentrated under reduced pressure. The residue was filtered through a pad of silica gel. The silyl ethers 7 were immediately subjected to FVP without further purification.

\section{(E)-1-Cyclopropyl-1-trimethylsilyloxycyclododec-3-ene (3)}

TMSO $\triangle$ The reaction of $(E)$-1-cyclopropylcyclododec-3-enol (6.8 g, $30.6 \mathrm{mmol})$ following the general procedure afforded $8.9 \mathrm{~g}(99 \%)$ of the title compound as a colorless oil. An analytical sample was distilled (Kugelrohr).

IR (film) 3085, 2924, 2852, 1462, 1445, 1358, 1300, 1250, 1207, 1181, $1156 \mathrm{~cm}^{-1}$; ${ }^{1} \mathrm{H}$ NMR (300 MHz, $\mathrm{CDCl}_{3}$ ) $\delta 5.45-5.36(\mathrm{~m}, 2 \mathrm{H}), 2.22-2.10(\mathrm{~m}, 2 \mathrm{H}), 2.08-1.99(\mathrm{~m}, 2 \mathrm{H}), 1.58-1.18(\mathrm{~m}, 14 \mathrm{H}), 0.89$ $(\mathrm{m}, 1 \mathrm{H}), 0.38-0.19(\mathrm{~m}, 4 \mathrm{H}), 0.01(\mathrm{~s}, 9 \mathrm{H}) ;{ }^{13} \mathrm{C} \mathrm{NMR}\left(75 \mathrm{MHz}, \mathrm{CDCl}_{3}\right) \delta 133.9,126.6,77.4,43.6,38.0$, 33.3, 28.3, 27.2, 26.4, 25.2, 22.7, 20.9, 19.7, 2.9, 1.0, 0.5; MS m/z (rel intensity) 294 (5), 195 (4), 183 (5), 169 (100), 141 (9), 130 (3), 73 (75). HRMS (EI) calcd for $\mathrm{C}_{18} \mathrm{H}_{34} \mathrm{OSi} m / z$ 294.2379, found $m / z$ 294.2377. Anal. Calcd for $\mathrm{C}_{18} \mathrm{H}_{34} \mathrm{OSi}$ (294.55): C, 73.40; H, 11.63. Found: C, 73.52; H, 11.70.

\section{(E)-1-Cyclopropyl-1-trimethylsilyloxycyclotridec-3-ene (7b)}

TMSO $\triangle$ The reaction of $(E)$-1-cyclopropylcyclotridec-3-enol (2.4 g, $10.2 \mathrm{mmol})$ following the general procedure afforded $3.1 \mathrm{~g} \mathrm{(99 \% )}$ ) of the title compound as a colorless oil (GC purity > 99\%). IR (film) 3084, 2931, 2859, 1461, 1446, 1249, 1072, 974, $838 \mathrm{~cm}^{-1}$; ${ }^{1} \mathrm{H}$ NMR (300 MHz, $\left.\mathrm{CDCl}_{3}\right) \delta 5.52-5.30(\mathrm{~m}, 2 \mathrm{H}), 2.27-2.10(\mathrm{~m}, 2 \mathrm{H}), 2.04-1.93(\mathrm{~m}, 2 \mathrm{H}), 1.68-1.17(\mathrm{~m}, 16 \mathrm{H})$, $1.02-0.87(\mathrm{~m}, 1 \mathrm{H}), 0.38-0.27(\mathrm{~m}, 4 \mathrm{H}), 0.02(\mathrm{~s}, 9 \mathrm{H}) ;{ }^{13} \mathrm{C} \mathrm{NMR}\left(75 \mathrm{MHz}, \mathrm{CDCl}_{3}\right) \delta 133.9,127.2,77.1$, 43.6, 38.6, 32.4, 27.8, 27.6, 27.5, 25.1, 25.0, 24.7, 20.9, 19.8, 2.8, 0.8, 0.7; MS m/z (rel intensity) 308 (5), 183 (4), 169 (100), 143 (9), 73 (90). HRMS (EI) calcd for $\mathrm{C}_{19} \mathrm{H}_{36} \mathrm{OSi} \mathrm{m} / \mathrm{z}$ 308.2535, found $\mathrm{m} / \mathrm{z} 308.2534$.

\section{(Z)-1-Cyclopropyl-1-trimethylsilyloxycyclooct-3-ene (7c)}

TMSO $\triangle$ The reaction of (Z)-1-cyclopropylcyclooct-3-enol $(3.8 \mathrm{~g}, 22.8 \mathrm{mmol})$ following the general procedure afforded $5.4 \mathrm{~g}(99 \%)$ of the title compound as a colorless oil (GC purity $>98 \%$ ). IR (film) 3084, 3018, 2932, 2857, 1465, 1446, 1250, 1071, $838 \mathrm{~cm}^{-1}$; ${ }^{1} \mathrm{H}$ NMR (300 MHz, $\left.\mathrm{CDCl}_{3}\right) \delta 5.81-5.51(\mathrm{~m}, 2 \mathrm{H}), 2.34(\mathrm{dd}, J=12.7,8.6 \mathrm{~Hz}, 1 \mathrm{H}), 2.19(\mathrm{dd}, J=12.7,7.4 \mathrm{~Hz}, 1 \mathrm{H}), 2.07-2.01$ $(\mathrm{m}, 2 \mathrm{H}), 1.63-1.35(\mathrm{~m}, 6 \mathrm{H}), 1.02-0.89(\mathrm{~m}, 1 \mathrm{H}), 0.39-0.27(\mathrm{~m}, 4 \mathrm{H}), 0.08(\mathrm{~s}, 9 \mathrm{H}) ;{ }^{13} \mathrm{C}$ NMR $(75 \mathrm{MHz}$, $\left.\mathrm{CDCl}_{3}\right) \delta 131.8,128.3,79.2,38.4,38.3,29.7,26.6,22.8,20.9,2.7,0.8,0.6$; MS m/z (rel intensity) 238 (2), 
195 (6), 169 (100), 141 (18), 73 (96). HRMS (EI) calcd for $\mathrm{C}_{14} \mathrm{H}_{26} \mathrm{OSi} \mathrm{m} / \mathrm{z}$ 238.1753, found $\mathrm{m} / \mathrm{z}$ 238.1751.

\section{(E)-1-(1-Methylcyclopropyl)-1-trimethylsilyloxycyclododec-3-ene (7d)}

TMSO $\searrow$ The reaction of (E)-1-(1-methylcyclopropyl)cyclododec-3-enol (2.7 g, $11.4 \mathrm{mmol})$ following the general procedure afforded $3.5 \mathrm{~g} \mathrm{(99 \% )}$ of the title compound as a colorless oil (GC purity $>99 \%)$.

IR (film) 3081, 2925, 2851, 1459, 1379, 1249, 1073, 980, 837; ${ }^{1} \mathrm{H}$ NMR (300 MHz, $\left.\mathrm{CDCl}_{3}\right) \delta$ 5.59-5.47 (m, 2H), 2.24-2.02 (m, 4H), 1.62-1.22 (m, 14H), $1.08(\mathrm{~s}, 3 \mathrm{H}), 0.68-0.56(\mathrm{~m}, 2 \mathrm{H}), 0.24-0.20$ $(\mathrm{m}, 1 \mathrm{H}), 0.14-0.09(\mathrm{~m}, 1 \mathrm{H}), 0.14(\mathrm{~s}, 9 \mathrm{H}) ;{ }^{13} \mathrm{C} \mathrm{NMR}\left(75 \mathrm{MHz}, \mathrm{CDCl}_{3}\right) \delta 134.0,126.5,80.2,39.0,36.0$, 33.5, 28.7, 27.1, 26.4, 25.2, 24.5, 22.7, 22.0, 20.0, 11.7, 10.2, 2.9; MS m/z (rel intensity) 308 (3), 253 (9), 183 (33), 169 (26), 144 (18), 73 (100). HRMS (EI) calcd for $\mathrm{C}_{19} \mathrm{H}_{36} \mathrm{OSi} \mathrm{m} / \mathrm{z}$ 308.2535, found $\mathrm{m} / \mathrm{z}$ 308.2538 .

\section{(E)-1-(cis-2-Methylcyclopropyl)-1-trimethylsilyloxycyclododec-3-ene (7e)}

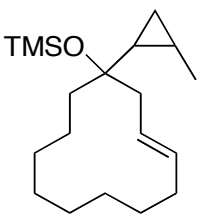

The reaction of $(E)-1$-(cis-2-methylcyclopropyl)cyclododec-3-enol (2.6 g, $11.0 \mathrm{mmol})$ following the general procedure afforded $3.3 \mathrm{~g}(98 \%)$ of the title compound as a colorless oil (GC purity > 99\%).

IR (film) 3069, 2928, 2852, 1461, 1446, 1250, 1091, 1067, 977, $837 \mathrm{~cm}^{-1} ;{ }^{1} \mathrm{H}$ NMR (300 $\left.\mathrm{MHz}, \mathrm{CDCl}_{3}\right) \delta 5.62-5.41(\mathrm{~m}, 2 \mathrm{H}), 2.42-2.34(\mathrm{~m}, 2 \mathrm{H}), 2.16-2.02(\mathrm{~m}, 2 \mathrm{H}), 1.64-1.23(\mathrm{~m}, 14 \mathrm{H}), 1.20(\mathrm{~d}, J$ $=6.5,3 \mathrm{H}), 0.92-0.82(\mathrm{~m}, 1 \mathrm{H}), 0.81-0.70(\mathrm{~m}, 1 \mathrm{H}), 0.51(\mathrm{td}, J=12.6,3.9,1 \mathrm{H}), 0.32-0.18(\mathrm{~m}, 1 \mathrm{H}), 0.12(\mathrm{~s}$, $9 \mathrm{H}) ;{ }^{13} \mathrm{C}$ NMR $\left(75 \mathrm{MHz}, \mathrm{CDCl}_{3}\right) \delta 133.7,126.9,78.1,46.3,39.7,32.6,27.5,27.1,26.8,24.9$, 24.6, 24.5, 19.6, 13.4, 10.8, 7.6, 1.3; MS m/z (rel intensity) 308 (4), 183 (52), 169 (8), 155 (15), 73 (100). HRMS (EI) calcd for $\mathrm{C}_{19} \mathrm{H}_{36} \mathrm{OSi} \mathrm{m} / \mathrm{z} 308.2535$, found $\mathrm{m} / z$ 308.2537.

\section{(E)-1-(trans-2-Methylcyclopropyl)-1-trimethylsilyloxycyclododec-3-ene}

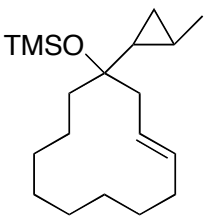

The reaction of (E)-1-(trans-2-methylcyclopropyl)cyclododec-3-enol (1.8 g, $7.6 \mathrm{mmol})$ following the general procedure afforded $2.3 \mathrm{~g} \mathrm{(97 \% )} \mathrm{of} \mathrm{the} \mathrm{title} \mathrm{compound} \mathrm{as} \mathrm{a} \mathrm{colorless} \mathrm{oil}$ (GC purity > 98\%).

IR (film) 3068, 2925, 2850, 1461, 1446, 1250, 1089, 1067, 977, $837 \mathrm{~cm}^{-1}$; ${ }^{1} \mathrm{H}$ NMR (300 $\left.\mathrm{MHz}, \mathrm{CDCl}_{3}\right) \delta 5.62-5.35(\mathrm{~m}, 2 \mathrm{H}), 2.40-2.32(\mathrm{~m}, 2 \mathrm{H}), 2.15-2.08(\mathrm{~m}, 2 \mathrm{H}), 1.66-1.19(\mathrm{~m}, 14 \mathrm{H}), 1.21(\mathrm{~d}, J$ $=6.6,3 \mathrm{H}), 0.86-0.67(\mathrm{~m}, 2 \mathrm{H}), 0.63-0.47(\mathrm{~m}, 1 \mathrm{H}), 0.29-0.11(\mathrm{~m}, 1 \mathrm{H}), 0.09(\mathrm{~s}, 9 \mathrm{H}) ;{ }^{13} \mathrm{C} \mathrm{NMR}(75 \mathrm{MHz}$, $\left.\mathrm{CDCl}_{3}\right) \delta 133.9,127.0,78.3,44.8,39.4,33.2,28.7,27.1,26.4,24.6,24.2,22.6,20.6,13.4,10.7,7.2,3.1$; MS m/z (rel intensity) 308 (3), 183 (48), 169 (9), 155 (17), 73 (100). HRMS (EI) calcd for $\mathrm{C}_{19} \mathrm{H}_{36} \mathrm{OSi} \mathrm{m} / z$ 308.2535 , found $m / z$ 308.2533. 
(E)-1-(2,2-Dimethylcyclopropyl)-1-trimethylsilyloxycyclododec-3-ene (7f)

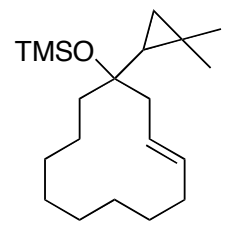

The reaction of $(E)$-1-(2,2-dimethylcyclopropyl)cyclododec-3-enol (1.3 g, $5.2 \mathrm{mmol})$ following the general procedure afforded $1.7 \mathrm{~g}(99 \%)$ of the title compound as a colorless oil (GC purity > 99\%).

IR (film) 2926, 2863, 1461, 1446, 1372, 1250, 1080, $981 \mathrm{~cm}^{-1} ;{ }^{1} \mathrm{H}$ NMR (300 MHz, $\left.\mathrm{CDCl}_{3}\right)$ $\delta 5.61-5.45(\mathrm{~m}, 2 \mathrm{H}), 2.35(\mathrm{~d}, J=5.7 \mathrm{~Hz}, 2 \mathrm{H}), 2.16-2.06(\mathrm{~m}, 2 \mathrm{H}), 1.61-1.23(\mathrm{~m}, 14 \mathrm{H}), 1.22(\mathrm{~s}, 3 \mathrm{H}), 1.02$ $(\mathrm{s}, 3 \mathrm{H}), 0.66(\mathrm{dd}, J=9.0,6.2 \mathrm{~Hz}, 1 \mathrm{H}), 0.41(\mathrm{dd}, J=6.2,3.6 \mathrm{~Hz}, 1 \mathrm{H}), 0.25(\mathrm{dd}, J=9.0,3.6 \mathrm{~Hz}, 1 \mathrm{H}), 0.13$ $(\mathrm{s}, 9 \mathrm{H}) ;{ }^{13} \mathrm{C}$ NMR $\left(75 \mathrm{MHz}, \mathrm{CDCl}_{3}\right) \delta 133.7,127.0,78.7,44.7,39.3,33.2,32.9,29.3,28.2,27.1,26.5$, 25.1, 24.6, 20.4, 19.7, 16.1, 15.2, 1.3; MS m/z (rel intensity) 322 (11), 279 (8), 197 (16), 169 (12), 141 (9), 107 (6), 91 (10), 73 (100). HRMS (EI) calcd for $\mathrm{C}_{20} \mathrm{H}_{38} \mathrm{OSi} \mathrm{m} / z$ 322.2692, found $\mathrm{m} / z, 322.2691$.

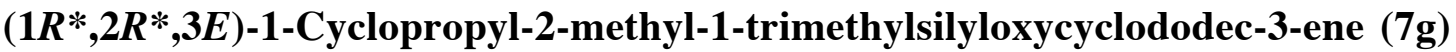

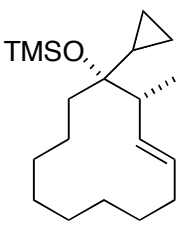

The reaction of $\left(1 R^{*}, 2 R^{*}, 3 E\right)$-1-cyclopropyl-2-methylcyclododec-3-enol (1.8 g, $\left.7.6 \mathrm{mmol}\right)$ following the general procedure afforded $2.0 \mathrm{~g}(87 \%)$ of the title compound as a colorless oil (GC purity > 97\%).

IR (film) 3085, 3005, 2935, 2864, 1462, 1364, 1249, 1167, 1072, 966, $838 \mathrm{~cm}^{-1} ;{ }^{1} \mathrm{H}$ NMR $\left(300 \mathrm{MHz}, \mathrm{CDCl}_{3}\right) \delta 5.47-5.34(\mathrm{~m}, 1 \mathrm{H}), 5.18-5.04(\mathrm{~m}, 1 \mathrm{H}), 2.96-2.86(\mathrm{~m}, 1 \mathrm{H}), 2.52-2.39(\mathrm{~m}, 1 \mathrm{H})$, $1.89-1.77(\mathrm{~m}, 1 \mathrm{H}) 1.51-1.06(\mathrm{~m}, 14 \mathrm{H}), 0.99(\mathrm{~d}, J=6.9 \mathrm{~Hz}, 3 \mathrm{H}), 0.59-0.20(\mathrm{~m}, 5 \mathrm{H}), 0.09(\mathrm{~s}, 9 \mathrm{H}) ;{ }^{13} \mathrm{C}$ NMR $\left(75 \mathrm{MHz} \mathrm{CDCl}_{3}\right) \delta 133.4,129.5,78.8,40.9,34.8,27.2,25.7,24.5,24.1,22.4,22.3,18.1,16.1,16.0$, 2.5, 1.8, 1.3; MS m/z (rel intensity) 308 (3), 183 (8), 169 (83), 141 (10), 73 (100). HRMS (EI) calcd for $\mathrm{C}_{19} \mathrm{H}_{36} \mathrm{OSi} \mathrm{m} / \mathrm{z} 308.2535$, found $\mathrm{m} / \mathrm{z} 308.2535$.

\section{General procedure for the thermal three-carbon ring expansion by FVP. ${ }^{5,6}$}

The FVP-device consists of an electrically heatable tube furnace (one meter-long), a condenser unit with a cooling trap at the outlet side and a kugelrohr oven as the evaporation unit at the inlet side. A quartz tube (110 cm long, $2.5 \mathrm{~cm}$ i. d., no fillings were used), which fitted into the furnace, was connected to a trap (cooled with liquid $\mathrm{N}_{2}$ ) on one side and to a bulb placed in the kugelrohr oven on the other. The starting material was placed in the bulb equipped with a capillary inlet device for the inert flow gas (a flow of $\mathrm{N}_{2}$ was adjusted from $0.8-1.4 \mathrm{~L} / \mathrm{h}$ ) and a magnetic stirrer. After evacuation of the apparatus with a highvacuum oil pump $\left(2-4 \times 10^{-2}\right.$ mbar $)$ the starting material was distilled directly through the preheated reactor tube $(1-3 \mathrm{~g} / \mathrm{h})$. After all of the starting material had been distilled, the apparatus was vented and the frozen products were transferred to a bulb using $\mathrm{Et}_{2} \mathrm{O}$ as solvent. The resulting solution was dried over anhydrous $\mathrm{MgSO}_{4}$ and evaporated under reduced pressure. The residue was dissolved in EtOH and treated with $1 \%$ $\mathrm{HCl}$ to cleave the TMS-group. Workup in the usual manner followed by flash chromatography on silica gel (1-10\% EtOAc in hexane) provided analytically pure compounds. 
(E)-Cyclopentadec-6-enone (5) and 5-Vinylcyclotridecanone (6)

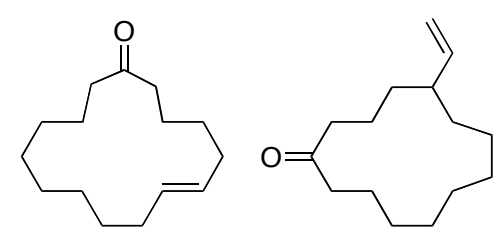

FVP of (E)-1-cyclopropyl-1-trimethylsilyloxycyclododec-3-ene (3) (4.60 g, $15.6 \mathrm{mmol})$ at $530{ }^{\circ} \mathrm{C}$ following the general procedure afforded $2.43 \mathrm{~g}$ (70\%) of $\mathbf{5}$ and $0.35 \mathrm{~g}(10 \%)$ of $\mathbf{6}$. Physical and spectral data of $\mathbf{5}$ are in accordance with literature data. ${ }^{7}$

Data of 5: IR (film) 2930, 2857, 1711, 1460, 1444, 1364, 1251, 1070, $976 \mathrm{~cm}^{-1}$; ${ }^{1} \mathrm{H}$ NMR (600 MHz, $\left.\mathrm{CDCl}_{3}\right) \delta 5.37-5.27(\mathrm{~m}, 2 \mathrm{H}), 2.38(\mathrm{t}, J=6.7 \mathrm{~Hz}, 2 \mathrm{H}), 2.34(\mathrm{t}, J=8.1 \mathrm{~Hz}, 2 \mathrm{H}), 2.08-2.02(\mathrm{~m}, 4 \mathrm{H})$, $1.65-1.56(\mathrm{~m}, 4 \mathrm{H}), 1.46-1.41(\mathrm{~m}, 2 \mathrm{H}), 1.40-1.33(\mathrm{~m}, 2 \mathrm{H}), 1.32-1.20(\mathrm{~m}, 8 \mathrm{H}) ;{ }^{13} \mathrm{C} \mathrm{NMR}(150 \mathrm{MHz}$, $\left.\mathrm{CDCl}_{3}\right) \delta 212.1,131.8,130.6,42.7,42.4,31.71,31.65,28.4,28.2,27.23,27.20,27.19,26.5,23.8,23.7$; MS $m / z$ (rel intensity) 222 (49), 179 (7), 165 (17), 151 (12), 137 (15), 123 (19), 109 (44), 98 (82), 81 (86), 67 (96), 55 (100). Anal. Calcd for $\mathrm{C}_{15} \mathrm{H}_{26} \mathrm{O}$ (222.37): C, 81.02; H, 11.78. Found: C, 80.88; H, 11.90.

Data of 6: IR (film) 3078, 2934, 2861, 1706, 1462, 1444, 1420, 1365, 1248, 1216. ${ }^{1} \mathrm{H}$ NMR (600 MHz, $\left.\mathrm{CDCl}_{3}\right) \delta 5.59(\mathrm{ddd}, J=17.0,10.2,7.3 \mathrm{~Hz}, 1 \mathrm{H}), 5.97(\mathrm{dd}, J=17.0,1.2 \mathrm{~Hz}, 1 \mathrm{H}), 4.94(\mathrm{dd}, J=10.2,1.2 \mathrm{~Hz}$, $1 \mathrm{H}), 2.53(\mathrm{ddd}, J=15.5,9.3,3.4 \mathrm{~Hz}, 1 \mathrm{H}), 2.44(\mathrm{td}, J=8.0,4.1 \mathrm{~Hz}, 2 \mathrm{H}), 2.34(\mathrm{ddd}, J=15.5,8.3,4.5 \mathrm{~Hz}$, $1 \mathrm{H}), 1.99(\mathrm{~m}, 1 \mathrm{H}), 1.78-1.53(\mathrm{~m}, 4 \mathrm{H}), 1.44-1.22(\mathrm{~m}, 14 \mathrm{H}) ;{ }^{13} \mathrm{C} \mathrm{NMR}\left(150 \mathrm{MHz}, \mathrm{CDCl}_{3}\right) \delta 212.9,143.6$, 113.7, 42.2 , 42.1 40.3, 32.5, 31.2, 26.7, 26.04, 26.00, 25.1, 23.5, 23.4, 21.1; HRMS (EI) for $\mathrm{C}_{15} \mathrm{H}_{26} \mathrm{O}$ : calcd 222.1984; found 222.1989. Anal. Calcd for $\mathrm{C}_{15} \mathrm{H}_{26} \mathrm{O}$ (222.37): C, 81.02; H, 11.78. Found: C, 81.19; H, 11.99.

\section{(Z)-Cyclopentadec-6-enone (8a)}

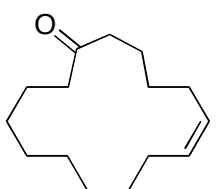

FVP of (Z)-1-cyclopropyl-1-trimethylsilyloxycyclododec-3-ene (7a) (2.53 g, $8.6 \mathrm{mmol})$ at $530{ }^{\circ} \mathrm{C}$ following the general procedure afforded $1.32 \mathrm{~g}(69 \%)$ of the title compound as a colorless oil. Physical and spectral data are in accordance with literature data. ${ }^{7}$ GC/MS analysis provided evidence for the presence of 5-vinylcyclotridecanone (6) (8\%).

IR (film) 2928, 2857, 1712, 1461, 1443, 1350, 1246, 1070, $971 \mathrm{~cm}^{-1} ;{ }^{1} \mathrm{H}$ NMR (300 MHz, $\left.\mathrm{CDCl}_{3}\right) \delta$ 5.36-5.27 (m, 2H), 2.45-2.32 (m, 4H), 2.09-1.99 (m, 4H), 1.68-1.55 (m, 4H), 1.46-1.17 (m, 12H); ${ }^{13} \mathrm{C}$ NMR $\left(75 \mathrm{MHz}, \mathrm{CDCl}_{3}\right) \delta 212.5,130.6,129.4,42.9,41.1,28.9,28.1,27.4,27.3,27.1,27.0,26.5,26.2$, 24.0, 23.0; MS m/z (rel intensity) 222 (45), 193 (5), 179 (6), 165 (12), 151 (12), 137 (15), 125 (20), 111 (46), 98 (78), 81 (83), 67 (100). Anal. Calcd for $\mathrm{C}_{15} \mathrm{H}_{26} \mathrm{O}$ (222.37): C, 81.02; H, 11.78. Found: C, 80.91; $\mathrm{H}, 11.83$.

\section{(E)-Cyclohexadec-6-enone (8b)}

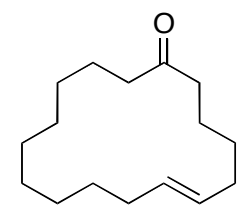

FVP of (E)-1-cyclopropyl-1-trimethylsilyloxycyclotridec-3-ene (7b) (1.65 g, $5.3 \mathrm{mmol})$ at $530{ }^{\circ} \mathrm{C}$ following the general procedure afforded $0.90 \mathrm{~g}(71 \%)$ of the title compound as a colorless oil. ${ }^{1} \mathrm{H}$ NMR of the crude product provided evidence for the presence of a minor amount of 5-vinylcyclotetradecanone (ca. 10\%).

IR (film) 2927, 2854, 1712, 1459, 1447, 1368, 1261, $968 \mathrm{~cm}^{-1} ;{ }^{1} \mathrm{H}$ NMR (300 MHz, $\left.\mathrm{CDCl}_{3}\right) \delta 5.39-5.29$ (m, 2H), 2.43 (t, $J=6.3 \mathrm{~Hz}, 2 \mathrm{H}), 2.38-2.32$ (m, 2H), 2.08-2.00 (m, 4H), 1.69-1.53 (m, 4H), 1.46-1.15 (m, 
$14 \mathrm{H}) ;{ }^{13} \mathrm{C} \mathrm{NMR}\left(75 \mathrm{MHz}, \mathrm{CDCl}_{3}\right) \delta 212.5,131.8,130.2,43.1,41.1,32.2,32.0,28.6,28.5,28.0,27.7$, 27.6, 26.9, 26.5, 24.1, 22.8; MS m/z (rel intensity) 236 (23), 152 (5), 137 (7), 123 (9), 111 (14), 95 (48), 81 (63), 67 (82), 55 (100). Anal. Calcd for $\mathrm{C}_{16} \mathrm{H}_{28} \mathrm{O}$ (236.40): C, 81.29; H, 11.93. Found: C, 81.23; H, 11.89.

\section{(Z)-Cycloundec-6-enone $(8 \mathrm{c})^{8}$}

o (Z)-1-Cyclopropyl-1-trimethylsilyloxycyclooct-3-ene (7c) $(2.10 \mathrm{~g}, 8.8 \mathrm{mmol})$ was pyrolyzed at $560{ }^{\circ} \mathrm{C}$ to afford $1.30 \mathrm{~g}$ (89\% recovery) of crude product. Flash chromatography of the hydrolyzed crude product (silica gel, $2 \%$ ethyl acetate in hexanes) afforded a fraction $(0.88 \mathrm{~g})$ containing the title compound accompanied with two unidentified side products in a 2:1:1 ratio. Hydrogenation of this mixture in the presence of $\mathrm{Pd} / \mathrm{C}$ and co-injection (GC/MS) with an authentic sample of cycloundecanone (purchased from Fluka) provided evidence for the initial formation of the title compound.

\section{(E)-2-Methylcyclopentadec-6-enone (8d)}

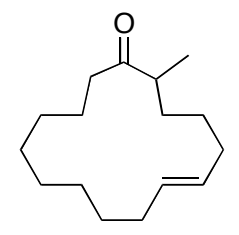

FVP of (E)-1-(1-methylcyclopropyl)-1-trimethylsilyloxycyclododec-3-ene (7d) (2.10 g, 6.8 mmol) at $520{ }^{\circ} \mathrm{C}$ following the general procedure afforded $1.55 \mathrm{~g}(72 \%)$ of the title compound as a colorless oil.

IR (film) 2929, 2855, 1711, 1459, 1447, 1370, $968 \mathrm{~cm}^{-1} ;{ }^{1} \mathrm{H} \mathrm{NMR}\left(300 \mathrm{MHz}, \mathrm{CDCl}_{3}\right) \delta$ 5.35-5.29 (m, 2H), 2.56-2.48 (m, 1H), $2.45(\mathrm{dd}, J=7.6,6.1 \mathrm{~Hz}, 1 \mathrm{H}), 2.32-2.22(\mathrm{~m}, 1 \mathrm{H}), 2.14-1.90$ (m, $4 \mathrm{H}), 1.72-1.62(\mathrm{~m}, 2 \mathrm{H}), 1.56-1.42(\mathrm{~m}, 2 \mathrm{H}), 1.41-1.17(\mathrm{~m}, 12 \mathrm{H}), 1.03(\mathrm{~d}, J=6.8 \mathrm{~Hz}, 3 \mathrm{H}) ;{ }^{13} \mathrm{C}$ NMR $(75$ $\left.\mathrm{MHz}, \mathrm{CDCl}_{3}\right) \delta 215.4,131.7,130.6,46.2$, 40.4, 31.7, 31.6, 31.3, 27.9, 27.5, 27.4, 27.3, 26.8, 26.6, 23.1, 14.9; MS m/z (rel intensity) 236 (12), 165 (5), 151 (4), 139 (7), 123 (9), 109 (26), 95 (47), 81 (60), 67 (69), 55 (100). Anal. Calcd for $\mathrm{C}_{16} \mathrm{H}_{28} \mathrm{O}$ (236.40): C, 81.29; H, 11.93. Found: C, 81.19; H, 11.91.

\section{(E)-4-Methylcyclopentadec-6-enone (8e)}

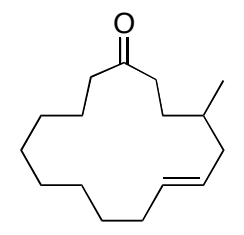

FVP of (E)-1-(cis-2-methylcyclopropyl)-1-trimethylsilyloxycyclododec-3-ene (7e) $(1.80 \mathrm{~g}$, $5.8 \mathrm{mmol})$ at $520{ }^{\circ} \mathrm{C}$ following the general procedure afforded $0.55 \mathrm{~g}(41 \%)$ of the title compound as a colorless oil. Alternatively, FVP of the diastereoisomeric (E)-1-(trans-2methylcyclopropyl)-1-trimethylsilyloxycyclododec-3-ene $(1.15 \mathrm{~g}, 3.7 \mathrm{mmol})$ at the same temperature afforded $0.35 \mathrm{~g}(41 \%)$ of the title compound as a colorless oil.

IR (film) 2928, 2856, 1708, 1458, $970 \mathrm{~cm}^{-1} ;{ }^{1} \mathrm{H}$ NMR (300 MHz, $\mathrm{CDCl}_{3}$ ) $\delta 5.37-5.32$ (m, 2H), 2.44-2.32 $(\mathrm{m}, 4 \mathrm{H}), 2.17-1.97(\mathrm{~m}, 4 \mathrm{H}), 1.88-1.13(\mathrm{~m}, 15 \mathrm{H}), 0.94(\mathrm{~d}, J=6.7 \mathrm{~Hz}, 3 \mathrm{H}) ;{ }^{13} \mathrm{C} \mathrm{NMR}\left(75 \mathrm{MHz}, \mathrm{CDCl}_{3}\right) \delta$ 213.4, 132.2, 129.3, 42.7, 40.4, 40.1, 32.9, 31.7, 30.2, 28.2, 27.1, 27.1, 27.2, 26.5, 23.7, 20.3; MS m/z (rel intensity) 236 (12), 151 (4), 136 (5), 125 (6), 109 (10), 97 (30), 81 (51), 67 (52), 55 (100). Anal. Calcd for $\mathrm{C}_{16} \mathrm{H}_{28} \mathrm{O}$ (236.40): C, 81.29; H, 11.93. Found: C, 81.25; H, 11.88. 
(E)-4,4-Dimethylcyclopentadec-6-enone (8f)

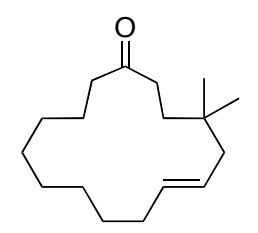

FVP of (E)-1-(2,2-dimethylcyclopropyl)-1-trimethylsilyloxycyclododec-3-ene (7f) $(1.10 \mathrm{~g}$, $3.4 \mathrm{mmol})$ at $510{ }^{\circ} \mathrm{C}$ following the general procedure afforded $0.43 \mathrm{~g}(50 \%)$ of the title compound as a colorless oil.

IR (film) 2929, 2858, 1710, 1459, 1447, 1385, 1366, 1120, $969 \mathrm{~cm}^{-1} ;{ }^{1} \mathrm{H}$ NMR $(300 \mathrm{MHz}$, $\left.\mathrm{CDCl}_{3}\right) \delta 5.41-5.35(\mathrm{~m}, 2 \mathrm{H}), 2.38(\mathrm{t}, J=6.5 \mathrm{~Hz}, 2 \mathrm{H}), 2.35-2.29(\mathrm{~m}, 2 \mathrm{H}), 2.05(\mathrm{q}, J=5.6 \mathrm{~Hz}, 2 \mathrm{H}), 1.94(\mathrm{~d}$, $J=5.8 \mathrm{~Hz}, 2 \mathrm{H}), 1.65-1.56(\mathrm{~m}, 2 \mathrm{H}), 1.47-1.41(\mathrm{~m}, 2 \mathrm{H}), 1.40-1.19(\mathrm{~m}, 10 \mathrm{H}), 0.91(\mathrm{~s}, 6 \mathrm{H}) ;{ }^{13} \mathrm{C}$ NMR $(75$ $\left.\mathrm{MHz}, \mathrm{CDCl}_{3}\right) \delta 213.5,133.0,127.5,45.3,42.7,37.6,34.8,32.8,31.8,28.3,27.6,27.0,26.8,26.7,26.1$, 23.7; MS m/z (rel intensity) 250 (6), 235 (4), 163 (7), 125 (68), 112 (46), 97 (37), 81 (43), 69 (55), 55 (100). Anal. Calcd for $\mathrm{C}_{17} \mathrm{H}_{30} \mathrm{O}$ (250.42): C, 81.54; H, 12.07. Found: C, 81.63; H, 12.15.

\section{(E)-5-Methylcyclopentadec-6-enone (8g)}

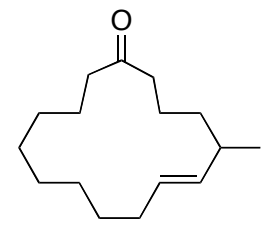

FVP of $\left(1 R^{*}, 2 R^{*}, 3 E\right)$-1-cyclopropyl-2-methyl-1-trimethylsilyloxycyclododec-3-ene (7g) $(1.80 \mathrm{~g}, 3.6 \mathrm{mmol})$ at $520{ }^{\circ} \mathrm{C}$ following the general procedure afforded $0.51 \mathrm{~g}(61 \%)$ of the title compound as a colorless oil.

IR (film) 2928, 2857, 1710, 1459, 1365, $970 \mathrm{~cm}^{-1} ;{ }^{1} \mathrm{H} \mathrm{NMR}\left(600 \mathrm{MHz}, \mathrm{CDCl}_{3}\right) \delta 5.30$ (ddd, $J=15.3,8.9,4.9 \mathrm{~Hz}, 1 \mathrm{H}), 5.16(\mathrm{ddd}, J=15.3,8.4,1.1 \mathrm{~Hz}, 1 \mathrm{H}), 2.44-2.32(\mathrm{~m}, 3 \mathrm{H}), 2.28-2.23(\mathrm{~m}$, 1H), 2.14-2.07 (m, 2H), 1.96-1.90 (m. 1H), 1.68-1.16 (m, 16H), $0.97(\mathrm{~d}, J=6.7 \mathrm{~Hz}, 3 \mathrm{H}) ;{ }^{13} \mathrm{C}$ NMR $(150$ $\left.\mathrm{MHz}, \mathrm{CDCl}_{3}\right) \delta 213.3,136.6,129.8,43.0,42.6,37.3,36.9,31.8,28.4,27.3,27.23,27.20,26.7,23.4,23.5$, 21.7; MS m/z (rel intensity) 250 (6), 235 (4), 163 (7), 125 (68), 112 (46), 97 (37), 81 (43), 69 (55), 55 (100). Anal. Calcd for $\mathrm{C}_{16} \mathrm{H}_{28} \mathrm{O}$ (236.40): C, 81.29; H, 11.93. Found: C, 81.23; H, 11.90.

\section{1-Cyclopropylcyclododec-11-en-1-one (11) and Bicyclo[10.3.0]pentadecan-2-one (12).}
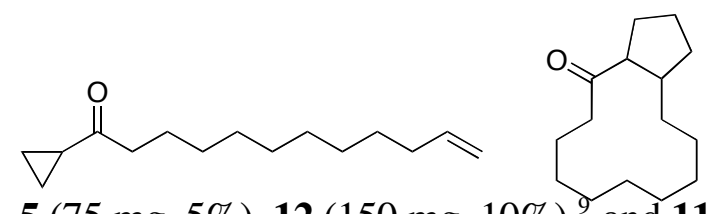

FVP of (E)-1-cyclopropylcyclododec-3-enol (10) $(1.50 \mathrm{~g}, 6.7$ mmol) at $530{ }^{\circ} \mathrm{C}$ following the general procedure, but without treatment of the crude product with $1 \% \mathrm{HCl}$ in $\mathrm{EtOH}$ afforded $5(75 \mathrm{mg}, 5 \%), \mathbf{1 2}(150 \mathrm{mg}, 10 \%)$, and $11(0.98 \mathrm{~g}, 65 \%)$ in that order.

Data of 11: IR (film) 3078, 3009, 2977, 2926, 2855, 1699, 1641, 1464, 1386, 1302, $1195 \mathrm{~cm}^{-1}$; ${ }^{1} \mathrm{H}$ NMR $\left(600 \mathrm{MHz}, \mathrm{CDCl}_{3}\right) \delta 5.87-5.74(\mathrm{~m}, 1 \mathrm{H}) 5.02-4.90(\mathrm{~m}, 2 \mathrm{H}), 2.53(\mathrm{t}, J=7.2 \mathrm{~Hz}, 2 \mathrm{H}), 2.03(\mathrm{q}, J=7.5 \mathrm{~Hz}$, $2 \mathrm{H}$ ), $1.92(\mathrm{~m}, 1 \mathrm{H}), 1.60$ (quint., $J=7.2 \mathrm{~Hz}, 2 \mathrm{H}), 1.37$ (quint., $J=7.5 \mathrm{~Hz}, 2 \mathrm{H}), 1.28-1.22(\mathrm{~m}, 10 \mathrm{H})$, 1.02-0.97 (m, 2H), 0.88-0.80 (m, 2H); $\left.{ }^{13} \mathrm{C} \mathrm{NMR} \mathrm{(150} \mathrm{MHz,} \mathrm{CDCl}_{3}\right) \delta 211.0,139.0,113.9,43.4,33.6$, 29.3, 29.1, 29.0, 28.8, 23.9, 20.1, 10.3; MS m/z (rel intensity) 222 (1), 151 (4), 137 (5), 97 (16), 84 (100), 69 (80). Anal. Calcd for $\mathrm{C}_{15} \mathrm{H}_{26} \mathrm{O}$ (222.37): C, 81.02; H, 11.78. Found: C, 80.96; H, 11.81.

Data of 12: IR (film) 2932, 2864, 1713, 1460, 1445, 1205, $\left.998 \mathrm{~cm}^{-1} ;{ }^{1} \mathrm{H} \mathrm{NMR} \mathrm{(600} \mathrm{MHz,} \mathrm{CDCl}_{3}\right) \delta$ $3.03-2.85(\mathrm{~m}, 2 \mathrm{H}), 2.23-1.95(\mathrm{~m}, 4 \mathrm{H}), 1.91-1.78(\mathrm{~m}, 1 \mathrm{H}), 1.77-1.53(\mathrm{~m}, 5 \mathrm{H}), 1.43-1.06(\mathrm{~m}, 14 \mathrm{H}) ;{ }^{13} \mathrm{C}$ NMR (150 MHz, $\left.\mathrm{CDCl}_{3}\right) \delta 213.3,55.3,43.3,40.8,31.8,27.3,26.32,26.31,25.9,25.0,24.1,24.0,22.9$, $22.3,21.6$. 


\section{References}

(1) Thies, R. W.; Bolesta, R. E., J. Org. Chem. 1976, 41, 1233.

(2) Heap, N.; Whitham, G. H., J. Chem. Soc. (B) 1966, 164.

(3) Crandall, J. K.; Banks, D. B.; Coler, R. A.; Watkins, R. J.; Arrington, J. P., J. Org. Chem. 1968, 33, 423.

(4) The use of benzene resulted in a variety of side products.

(5) Rüedi, G.; Nagel, M.; Hansen, H.-J., Org. Lett. 2003, 5, 2691.

(6) McNab, H., Contemp. Org. Synth. 1996, 3, 373.

(7) Fehr, C.; Galindo, J.; Etter, O.; Thommen, W., Angew. Chem., Int. Ed. 2002, 41, 4523.

(8) Zhang, W.; Dowd, P., Tetrahedron Lett. 1996, 37, 957.

(9) Only a single diastereoisomer was formed, but its relative configuration could not be assigned by ${ }^{1} \mathrm{H}$ NOE due to overlapping ${ }^{1} \mathrm{H}$ signals. 